(CASE REPORT)

\title{
A case report of Weil's disease
}

\author{
Sanjay Sud \\ Designation: Visiting Consultant Internal Medicine, Kamala Ray Hospital, 20, Kotrung, G.T. Road, Hindmotor, District - \\ Hooghly, PIN - 712 233, West Bengal, India.
}

International Journal of Biological and Pharmaceutical Sciences Archive, 2021, 01(01), 023-026

Publication history: Received on 03 January 2021; revised on 10 January 2021; accepted on 12 January 2021

\begin{abstract}
This study present the details of a case of classical Weil's Disease, which is a severe form of leptospirosis, presenting with symptoms of fever, jaundice, joint pain, haemorrhagic manifestations and renal failure. Leptospirosis is an uncommon but not rare health problem in a tropical country like India. Clinical manifestations along with the laboratory findings are elaborated, so that a differential diagnosis from other common ailments with similar manifestations can be considered in favour of the disease. An early empirical management on high clinical suspicion can help to avoid multiorgan dysfunction and mortality associated with this disease.
\end{abstract}

Keywords: Jaundice; leptospirosis; Renal failure; Hyperglycaemia; Myalgia; Arthralgia; Haemorrhagic manifestations; Zoonotic disease and Weil's disease.

\section{Introduction}

Leptospirosis is a blood infection caused by the Leptosira species, which are spirochetes belonging to the order Spirochaetales and the family Leptospiraceae. Signs and symptoms can range between none to mild (headaches, muscle pains, and fevers) to severe (bleeding in the lungs or meningitis). Weil's disease, the acute, severe form of leptospirosis, causes the infected person to become jaundiced (skin and eyes become yellow), develop renal failure, and bleed [1]. Bleeding from the lungs associated with leptospirosis is known as "severe pulmonary haemorrhage syndrome". More than 10 genetic types of Leptospira cause disease in humans. Both wild and domestic animals can spread the disease, most commonly rodents. The bacteria are spread to humans through animal urine, water and soil, contaminated with animal urine, coming into contact with the eyes, mouth, nose and breaks in the skin.

The differential diagnosis of leptospirosis depends on the epidemiology of acute febrile illnesses in a particular area. A high index of suspicion is needed in endemic areas, and leptospirosis must be considered when a patient presents with acute onset of fever, headache, myalgia and jaundice. However, in locations where dengue fever or malaria is also endemic, the differentiation may be very difficult because of similar clinical manifestations. Laboratory confirmation is crucial, especially when these diseases are occurring simultaneously during the rainy season [5]. Hence one of the reasons for presenting this case report is to increase the awareness regarding this zoonotic disease.

\section{Case report}

The patient was a 48 year old female, who used to share managing a small restaurant along with her husband, located near a suburban railway platform (in the suburbs of the metropolis of Kolkata, West Bengal, India). In general these local eateries have a very low hygienic standard and their surroundings too are very un-hygienic. Poor drainage and

\footnotetext{
${ }^{*}$ Corresponding author: Dr. Sanjay Sud

Designation: Visiting Consultant Internal Medicine, Kamala Ray Hospital, 20, Kotrung, G.T. Road, Hindmotor, District - Hooghly, PIN - 712 233, West Bengal, India.
}

Copyright (C) 2021 Author(s) retain the copyright of this article. This article is published under the terms of the Creative Commons Attribution Liscense 4.0. 
infestation of rodents in these places is very common. She presented in my clinic with high fever, joint pain, yellow discoloration of the eyes, anorexia, haematuria, nausea and vomiting. The history of onset of her symptoms started with fever, myalgia and joint pain, four days prior to her visit in my clinic. She did not have any significant past medical history and was not on any regular medications. On examination - icterus, discreet bilateral conjunctival hemorrhages, pyrexia $\left(103^{\circ}\right.$ Fahrenheit - axillary) and a dry tongue (dehydration) were noted. She had tachycardia (Pulse rate of 136 / minute), diffused bilateral coarse chest crepitations and a blood pressure of 156/96 mm of Hg. SpO2 was 97 \% in room air. She was advised hospitalization immediately. Supportive therapy with intravenous fluids, PPI and paracetamol was started.

The investigations done revealed the following:

Total Leucocyte count - 14,600/cu.mm.(Neutrophil 85\%),

Hb \% 9.0 gm/dl, Platelet count 3,13,000 / cu.mm., ESR 82 mm. Urea 92 mg/dl, Creatinine $3.97 \mathrm{mg} / \mathrm{dl}$,

CRP $191.3 \mathrm{mg} / \mathrm{L}$, Serum Sodium $126 \mathrm{mEq} / \mathrm{L}$, Serum Potassium $2.8 \mathrm{mEq} / \mathrm{L}$,

Total Bilirubin 13.07 mg/dl (Conjugated 11.08 mg/dl), ALT 28 IU/L, AST 35 IU/L,

Random blood Sugar was $216 \mathrm{mg} / \mathrm{dl}$, INR 1.2,

Urine RE showed Granular casts with 5 - 6 R.B.Cs per H.P.F.

U.S.G. of the abdomen revealed moderate Hepatomegaly and Splenomegaly.

Chest $\mathrm{X}$ ray showed diffused bilateral patchy pneumonitis (Fig. 1).

Immediately after admission she developed diffused petechia and haemoptysis and her icterus increased. There was severe arthralgia and myalgia with recurrence of fever as soon as the action of paracetamol waned off.

Sputum examination was negative for AFB, Gram stain showed presence of commensals.

Dengue NS 1 antigen and Dual Malaria antigen were negative.

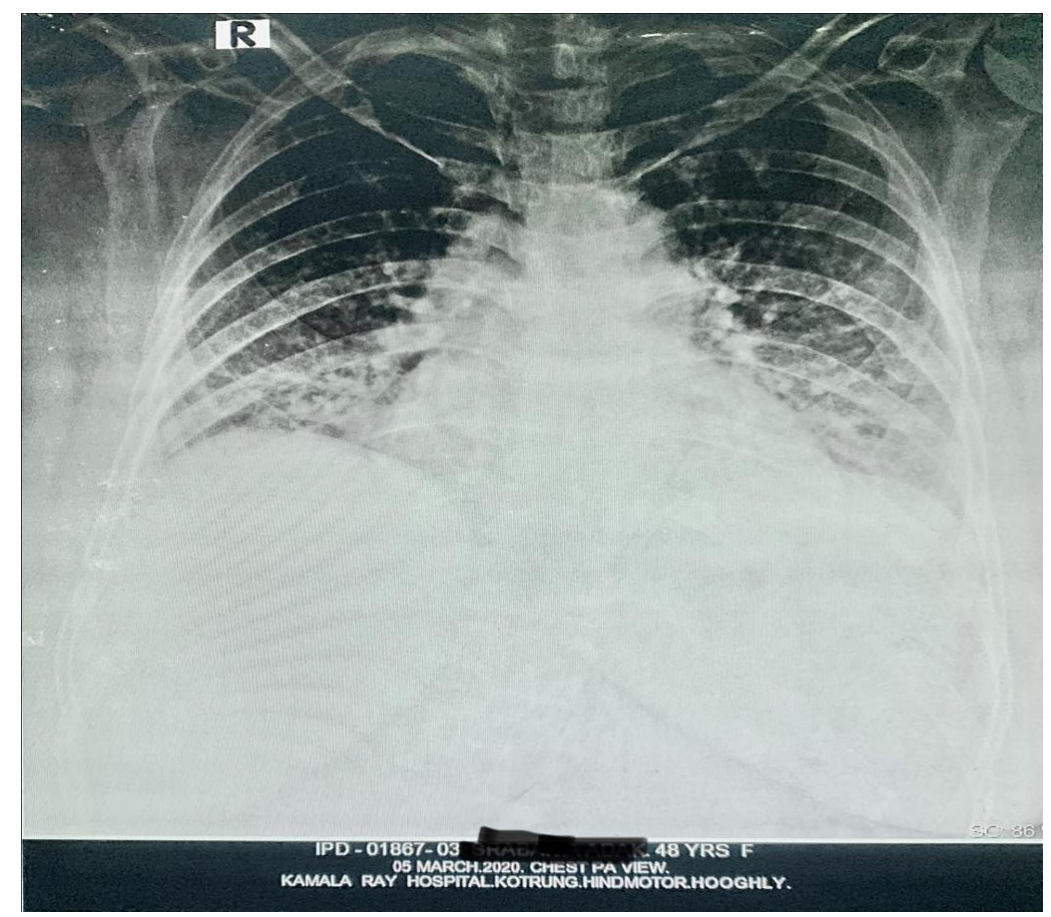

Figure 1. Chest X -ray

These clinical manifestations, history and working habitat of the patient led to a high suspicion of Leptospirosis. Hence Serum Leptospira Antibody IgM (by ELISA) was done.

The Leptospira antibody titre was 3.47 (reference range - Negative $<0.9$, Equivocal $0.9-1.1$ and Positive $>1.1$ ) 
After confirmation of the diagnosis as Weil's disease the patient was administered IV Ceftriaxone 2 gms OD and IV Doxycycline $200 \mathrm{mg}$ as loading dose, followed by $100 \mathrm{mg} 12$ hourly (both continued for 7 days) [1], along with supportive therapy like IV fluids, PPI, antipyretics etc. (Prior to receiving the Leptospira antibody titre report she was empirically put on IV Co-Amoxyclav 1.2 gms TID and IV Doxcycline $100 \mathrm{mg}$ BID).

There was an increase in the level of Total Bilirubin to $16.05 \mathrm{mg} / \mathrm{dl}$ on the third day after hospital admission. The other biochemical derangements (hyponatremia, hypokalemia, hyperglycaemia, raised creatinine and leukocytosis) slowly improved and came back to normal by the $7^{\text {th }}$ day of institutional treatment (Urea $28 \mathrm{mg} / \mathrm{dl}$, Creatinine $1.35 \mathrm{mg} / \mathrm{dl}$ and TLC 11,000 /cu.mm). Serum Bilirubin still remained $10.78 \mathrm{mg} / \mathrm{dl}$ on the $7^{\text {th }}$ day of indoor treatment initiation.

Clinically too patient was afebrile, had no joint pain, myalgia, haemoptysis or other haemorragic manifestations (petechiae) on the $7^{\text {th }}$ day after hospitalization. She had to be discharged with anti hypertensives (to be continued) along with oral Doxycycline (100 mg OD for 10 days).

On follow up at two weeks after her discharge from the hospital her Liver function tests were completely normal. Her anti-hypertensive medications could be tapered down and stopped within 6 weeks post discharge. She was able to return back to her normal routine of helping out her husband with running their restaurant, albeit with her being made aware about the best possible work and environmental hygiene.

\section{Discussion}

Leptospirosis is an acute and often severe infection that frequently affects the liver or other organs and is caused by the multiple serovars of Leptospira interrogans [1]. In 1885, Adolf Weil described the clinical hallmarks of this disease as an acute process characterized by splenomegaly, jaundice, and nephritis. With time, the designation Weil's disease came to signify severe leptospirosis characterized by diverse clinical findings, particularly fever, jaundice, acute renal injury, refractory shock, (though in this particular case the patient had hypertension needing management by antihypertensive medications) and hemorrhage (especially pulmonary hemorrhage) [2]. In general, clinical manifestation can be divided into two distinct clinical syndromes - $90 \%$ of patients present with mild anicteric febrile illness associated with influenza-like illness with headache and myalgia, 10\% are severely ill with jaundice and other manifestations (Weil's disease). In icteric leptospirosis (Weil's syndrome), persistent high fever and jaundice are usually associated with hepatic dysfunction, renal failure, hemorrhage and multi-organ failure (MOF). Hemorrhage can occur as petechiae, conjunctival hemorrhage and gastrointestinal hemorrhage. MOF is associated with a very high mortality. Myocarditis and hemorrhagic pulmonary infiltration are other complications, which may prove fatal [4]. Leptospirosis should be suspected on the basis of an appropriate exposure history combined with any of the infection's protean manifestations. A high index of suspicion prompting elicitation of a detailed exposure history is critical and guides confirmatory testing.

Weil's disease is suggested by elevated levels of blood urea and serum creatinine in conjunction with mixed conjugated and unconjugated hyperbilirubinemia with aminotransferase elevation to $<5$ times the upper limit of normal. When a patient is having jaundice, the bilirubin level is markedly raised but transaminase and alkaline phosphatase concentrations may not rise much beyond the upper limit of normal [1]. Jaundice is not associated with fulminant hepatic necrosis or hepatocellular damage, but rather with abnormal laboratory values. Hematologic abnormalities are variable but common. Leukocytosis (typical in severe disease) / leukopenia, hemolytic anemia, mild to moderate anemia, and thrombocytopenia is often noted. The anemia frequently observed in Weil's syndrome has been ascribed on clinical impression to blood loss, renal failure, and/or an ill-defined hemolytic process. However, hemolytic anemia associated with leptospirosis in animals is well documented and is due to hemolysins with phospholipase activity [6]. However, the prothrombin and activated partial thromboplastin times are not necessarily elevated in severe leptospirosis [1].

It is unlikely that the early nonspecific illness of leptospirosis will be diagnosed unless there is a clear suggestion of the diagnosis in some occupational or recreational exposure, or if there is an outbreak. Clinical clues that may suggest the diagnosis of leptospirosis over other causes of acute fever are disproportionate myalgia, jaundice, conjunctival suffusion, petechial rash and lymphocytic meningitis [3].

The IgM ELISA is particularly useful in making an early diagnosis, since it is positive as early as 2 days into the illness, a time when the clinical manifestations may be nonspecific, and it is extremely sensitive and specific (93\%) [2]. 


\section{Conclusion}

Weil's Disease, is a severe form of leptospirosis, presenting with symptoms of fever, jaundice, joint pain, haemorrhagic manifestations and renal failure. Leptospirosis is an uncommon but not rare health problem in a tropical country like India. The clinicians dealing with patients coming to them with the aforementioned symptoms need to be aware of this disease, so that they can consider it in the differential diagnosis and initiate early management for it, and avoid multiorgan dysfunction and mortality associated with this disease.

\section{Compliance with ethical standards}

\section{Acknowledgments}

I acknowledge to the academic suggestions of my junior colleague Dr. Sanjoy Banerjee DM (Gastroenterology) for proceeding with the Leptospira antibody test. Also I acknowledge to the co-operation of the Directors of Kamala Ray Hospital, Mr. Ananta Ray and Ms. Amrita Ray

\section{Disclosure of conflict of interest}

There are no conflicts of interest.

\section{Statement of informed consent}

The author certifies that he has obtained all appropriate patient consent forms. In the form the patient has given her consent for her images and other clinical information to be reported in the journal. The patient understands that her name and initials will not be published and due efforts will be made to conceal her identity, but anonymity cannot be guaranteed.

\section{References}

[1] Leptospirosis - J.F.P. Wagenaar, Marga GA. Goris In: Jameson JL, Fauci AS, Kasper DL, Hauser SL, Longo DL, Loscalzo J, editors. Harrison's Principles of Internal Medicine. 20 th edition. New York, The Mc-Graw Hill Companies. 2018; 1290 - 94.

[2] Susan SP. Leptospirosis. In: Papadakis MA, McPhee SJ, editors. Current Medical Diagnosis and Treatment. 52nd edition, USA: The Mc-Graw Hill Companies. 2013; 1477-8.

[3] Geoffrey MS, Timothy JC. Leptospirosis. In: Cook GC, Zumila AL, editors. Manson's Tropical Diseases. $22^{\text {nd }}$ edition, USA: Saunders. 2008; 1161-6.

[4] Dutta TK, Christopher M. Leptospiros - An Overview. Journal of Association of Physicians of India. 2005; 53:545-51.

[5] Dockrell DH, Sundar S, Angus BJ, Hobson RP. Leptospirosis. In: Colledge NR, Walker BR, Ralston SH, editors. Davidson's Principles \& Practice of Medicine. 21 st edition. Edinburgh: Churchill Livingstone. 2010; 331-2.

[6] Trowbridge AA, Green JB 3rd, Bonnett JD, Shohet SB, Ponnappa BD, McCombs WB 3rd. Hemolytic anemia associated with leptospirosis. Morphologic and lipid studies. American Journal of Clinical Pathology. 1981;76:493-8. 